\title{
Ultra-high accuracy optical testing: creating diffraction-limited short-wavelength optical systems
}

\author{
Kenneth A. Goldberg ${ }^{1}$, Patrick P. Naulleau ${ }^{1}$, Senajith B. Rekawa ${ }^{1}$, Paul E. Denham ${ }^{1}$, \\ J. Alexander Liddle ${ }^{1}$, Eric M. Gullikson ${ }^{1}$, Keith H. Jackson ${ }^{1}$, Erik H. Anderson ${ }^{1}$, John S. Taylor ${ }^{2}$, \\ Gary E. Sommargren², Henry N. Chapman², Donald W. Phillion², Michael Johnson², \\ Anton Barty ${ }^{2}$, Regina Soufli ${ }^{2}$, Eberhard A. Spiller ${ }^{2}$, Christopher C. Walton ${ }^{2}$, Saša Bajt ${ }^{2}$ \\ ${ }^{1}$ Center for X-Ray Optics, Lawrence Berkeley National Laboratory, Berkeley, CA 94720 \\ ${ }^{2}$ Lawrence Livermore National Laboratory, Livermore, California 94550
}

\begin{abstract}
Since 1993, research in the fabrication of extreme ultraviolet (EUV) optical imaging systems, conducted at Lawrence Berkeley National Laboratory (LBNL) and Lawrence Livermore National Laboratory (LLNL), has produced the highest resolution optical systems ever made. We have pioneered the development of ultra-high-accuracy optical testing and alignment methods, working at extreme ultraviolet wavelengths, and pushing wavefront-measuring interferometry into the 2-20-nm wavelength range $(60-600 \mathrm{eV})$. These coherent measurement techniques, including lateral shearing interferometry and phase-shifting point-diffraction interferometry (PS/PDI) have achieved RMS wavefront measurement accuracies of $0.5-1-\AA$ and better for primary aberration terms, enabling the creation of diffraction-limited EUV optics. The measurement accuracy is established using careful null-testing procedures, and has been verified repeatedly through high-resolution imaging. We believe these methods are broadly applicable to the advancement of short-wavelength optical systems including space telescopes, microscope objectives, projection lenses, synchrotron beamline optics, diffractive and holographic optics, and more.

Measurements have been performed on a tunable undulator beamline at LBNL's Advanced Light Source (ALS), optimized for high coherent flux; although many of these techniques should be adaptable to alternative ultraviolet, EUV, and soft $\mathrm{x}$-ray light sources. To date, we have measured nine prototype all-reflective EUV optical systems with NA values between 0.08 and 0.30 ( $f / 6.25$ to $f / 1.67)$. These projection-imaging lenses were created for the semiconductor industry's advanced research in EUV photolithography, a technology slated for introduction in 2009-13. This paper reviews the methods used and our program's accomplishments to date.
\end{abstract}

Keywords: extreme ultraviolet, EUV, optics, diffraction-limited, interferometry

\section{INTRODUCTION}

The recent development of high-efficiency, diffraction-limited optical systems for extreme ultraviolet (EUV) wavelengths could usher a new generation of space-astronomical telescopes with unprecedented angular resolutions. Designed with fabrication error tolerances five to ten times more stringent than the current state-of-the-art telescopes, these new optical systems are being created to meet the demands of the semiconductor industry, which uses photolithography to massproduce microprocessors, computer memory, and other advanced electronic components. According to current, industry expectations, ${ }^{1,2}$ by 2009-13, the semiconductor industry will use photolithography based on EUV light (13.5-nm wavelength, 92.1-eV photon energy). Diffraction-limited EUV optical systems have extraordinarily tight fabrication and alignment tolerances and are arguably the highest quality optical imaging systems ever created. In a spectral region characterized by high absorption in nearly every material, EUV multilayer mirror coatings with nearly $70 \%$ reflectivity are routinely created from (nominally) forty molybdenum/silicon $(\mathrm{Mo} / \mathrm{Si}$ ) layer pairs (each bi-layer is approximately $\lambda / 2,7$-nm thick).

Recently designed short-wavelength-imaging space telescopes have angular resolutions of approximately 0.5 arcsec. For example, the lens in the Chandra X-Ray Observatory ${ }^{3}$ uses nested, glancing-incidence mirrors, and operates at photon energies between 0.1 and $10 \mathrm{keV}$ (1.2- $\AA$ to $12-\mathrm{nm}$ wavelength). The Atmospheric Imaging Assembly (AIA) instrument on the Solar Dynamics Observatory (SDO), ${ }^{4}$ scheduled for launch in 2008, operates at a variety of EUV wavelengths 
using multilayer-coated, near-normal incidence mirrors. It is designed to capture full images of the Sun, 41 arcmin, across a detector with 4096 pixels; the angular resolution per pixel is thus 0.6 arcseconds. The fabrication tolerances for these optical systems (discussed in Section 2) are significantly less stringent that those of existing prototype all-reflective EUV lenses designed for photolithography.

Visible-light interferometry is essential for the development and fabrication of individual EUV mirror elements, and assembled systems; yet visible-light cannot probe the EUV resonant-reflective response of the multilayer-coated mirrors. With respect to reflectivity alone, EUV multilayer coatings can perform well with a few percent change in the multilayer $d$-spacing (under ideal conditions, reflectivity FWHM is approximately 5\%. ${ }^{5}$ ); however in a diffraction-limited EUV optic, there is a significant concern for the phase of the reflected wave, which is much more sensitive to thickness variations. (Near the optimized design, the approximate phase dependence is: $\Delta \phi$ [waves] $=-7.49 \Delta d / d .^{5}$ ) Systems are often designed with a coating-thickness gradient in order to maintain high reflectivity given the variation of angles of incidence within the system. Yet since the reflected wavefront phase is highly sensitive to the $d$-spacing variation and the angle of incidence, optical systems must be designed including both the surface shape and the multilayer contribution. Furthermore, some amount of substrate asphericity is required to accommodate the coating thickness variation. Until perfect control in multilayer deposition is established, EUV interferometry, which has already demonstrated 0.1-nm measurement accuracy, will be necessary to provide unique and unambiguous feedback about the performance of the system wavefront. Furthermore, since interferometry uses the light-wavelength as a length scale, EUV interferometry has an advantage of approximately forty-times shorter wavelength: $0.1 \mathrm{~nm}$ is $1 / 135^{\text {th }}$ of an EUV wave, and approximately $1 / 5000^{\text {th }}$ of a visiblelight wave - an extreme challenge to measure.

Until recently, EUV interferometry has been performed almost exclusively at synchrotron sources ${ }^{6-9}$ However high-power incoherent EUV sources based on laser discharge or laser-plasma interactions,${ }^{10}$ and coherent EUV sources based on highharmonic generation ${ }^{11}$ and laser amplification in discharge-created plasmas ${ }^{12}$ are all promising non-synchrotron candidate sources for EUV interferometry.

This paper describes visible-light and EUV interferometry techniques developed for the creation of diffraction-limited EUV optical systems, giving examples from recently fabricated lenses. The availability of these techniques could spur the creation of a new generation of higher angular resolution astronomical instruments.

\section{FABRICATION TOLERANCES}

Because the operational light-wavelength is the relevant length scale for setting the specifications on any optical system, fabrication tolerances for multi-element lenses designed for EUV wavelengths are proportionally smaller than visible-light and UV applications. For diffraction-limited EUV optics, specifications are set to ensure the completed system has imaging resolution close to the physical limit, with high-contrast, and high efficiency.

Aberrations span a continuum of spatial frequencies from the full-size of the mirror substrates down to the atomic scale. Lens-designers often separate the spatial-frequency domains by their respective behaviors into three broad categories: wavefront aberrations, roughness, and micro-roughness. System wavefront aberrations (typically spanning length scales of order $10 \mathrm{~cm}$ down to $5 \mathrm{~mm}$, depending on the pupil diameter) are defined by the low-spatial-frequency mirror-shape deformations and system misalignments, and must be limited to a fraction of a nanometer to maintain a high Strehl ratio. Owing to the short EUV wavelengths, mid-spatial-frequency aberrations (typically spanning length scales of $5 \mathrm{~mm}$ down to $5 \mu \mathrm{m}$ ) are a significant concern for maintaining high-imaging contrast. Also referred to as roughness, this aberration spatial-frequency-domain contains the components that scatter light to small angles within the pupil. At the highest spatialfrequency range, micro-roughness (typically spanning length scales below $5 \mu \mathrm{m}$ ) scatters light outside of the pupil and contributes to a loss in mirror-reflectivity and system throughput.

Table 1 shows the fabrication specifications for the AIA instrument ${ }^{13}$ compared with a commercial research prototype EUV lithography lens: the Micro-Exposure Tool (MET) ${ }^{14}$ Both systems have two near-normal-incidence reflective mirrors, one concave and one convex. The MET is designed for $5 \times$ demagnification and has fixed conjugate planes separated by $474 \mathrm{~mm}$. With an annular pupil, and a relatively high numerical aperture of $0.3 \mathrm{NA}, f / 1.67$, the MET achieves diffraction-limited imaging with a Rayleigh resolution $(0.61 \lambda / \mathrm{NA})$ of $27-\mathrm{nm}$ half-pitch, and a 12-nm upper-limit half-pitch with off-axis 
Table 1. Figure and finish specifications for two EUV optical systems [Refs. 13,14,16,17]

\begin{tabular}{ll|ll}
\multicolumn{1}{l}{ AIA Instrument (space telescope) } & \multicolumn{2}{l}{ MET (EUV lithography optics) } \\
\hline Goal & $\begin{array}{l}0.6 \text { arcsec } \\
\text { angular resolution }\end{array}$ & Goal & $\begin{array}{l}32-\mathrm{nm} \\
\text { half pitch }\end{array}$ \\
\hline slope error & $\leq 5 \mu \mathrm{rad}$ & slope error & $\begin{array}{l}0.3-1.0 \mu \mathrm{rad}, \\
\text { measured }\end{array}$ \\
\hline $\begin{array}{l}\text { roughness } \\
1 / f=(4 \mu \mathrm{m}, 4 \mathrm{~mm})\end{array}$ & $\begin{array}{l}\text { MSFR (mid spatial- } \\
\text { frequency roughness) }\end{array}$ \\
\hline $\begin{array}{l}\text { micro-roughness } \\
1 / f=(9 \mathrm{~nm}, 4 \mu \mathrm{m})\end{array}$ & $\leq 4.4 \AA$ & $\begin{array}{l}\text { HSFR (high spatial- } \\
\text { frequency roughness) }\end{array}$ & $\leq 1-2 \AA$
\end{tabular}

illumination. The AIA telescope lens has a focal length of $4.125 \mathrm{~mm}$, and an angular resolution of 0.6 arcsec per $12-\mu \mathrm{m}$ CCD detector pixel. While the AIA represents a state-of-the-art EUV telescope, is not designed for diffraction-limited performance, and for that reason its specifications are comparably looser.

Technologies that make the creation of diffraction-limited EUV optics possible include accurate aspherical mirror polishing and metrology, the creation of ultra-smooth surfaces with below 2- $\AA$ RMS roughness, the application of large-area gradedthickness multilayer coatings with angstrom-scale roughness and thickness controls, ${ }^{15}$ and angstrom-scale-accuracy visible-light and EUV interferometries. For more than ten years, the development of these optical fabrication technologies has been the focus of research at Lawrence Berkeley and Lawrence Livermore National Laboratories (LBNL, and LLNL respectively). Although the mechanical figuring and polishing of aspherical mirror substrates is performed by commercial companies using proprietary methods, research in metrology performed to assess mirror figure and finish, and to mount and house mirror assemblies, has been conducted by LLNL. For low- and mid-spatial frequencies, visible-light interferometry is used (see in Section 4). Mid-spatial-frequency roughness has been studied effectively with EUV scatterometry performed at LBNL. ${ }^{16,17}$ Measurements made on individual mirror substrates can be combined mathematically to predict imaging contrast for assembled optical systems. High-spatial-frequency roughness is typically measured with atomic-force microscopy (AFM). ${ }^{18}$

\section{TRACK RECORD}

Since 1996, nine different prototype, reflective EUV optics have been measured and aligned at LLNL (using visiblelight interferometry) and LBNL (using EUV interferometry), some on multiple occasions. Once beam-alignment has been established, individual measurements can be made with 5-30 seconds per exposure frame; measurement at multiple field points across an optic's field of view typically requires several hours to complete. Table 2 lists the three different types of prototype EUV optical systems we have measured, each incorporating aspherical mirror elements. Although their full pupil is annular, the Schwarszschild objectives were used in an off-axis configuration to generate a circular disk pupil. These optics served as a test bed for the fabrication and metrology techniques being developed for the more advanced four-mirror systems build for the Engineering Test Stand (ETS) at Sandia National Laboratories ${ }^{19}$ - two of those systems were fabricated and used. The most recent system is the MET optic, ${ }^{14}$ a small-field system with three times higher resolution than the ETS lenses.
Table 2. Three generations of EUV reflective optics measured with EUV interferometry.
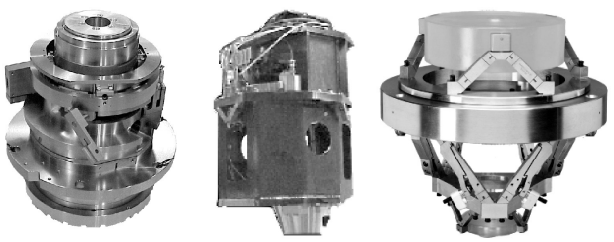

optic type Schwarzschild Engineering Micro Exposure Test Stand (ETS) Tool (MET)

\begin{tabular}{|cccc}
\hline wavelength & $13.4 \mathrm{~nm}$ & $13.4 \mathrm{~nm}$ & $13.5 \mathrm{~nm}$ \\
\hline magnification & $10 \times$ & $4 \times$ & $5 \times$ \\
\hline NA & $0.08-0.088$ & 0.1 & 0.3 , annular \\
\hline \# of mirrors & 2 & 4 & 2 \\
\hline track length $^{\mathrm{a}}$ & $31.5 \mathrm{~cm}$ & $108.5 \mathrm{~cm}$ & $47.42 \mathrm{~cm}$ \\
\hline field of view & $(100 \mu \mathrm{m})^{2}$ & $26 \times 1.5 \mathrm{~mm}$ & $3 \times 1 \mathrm{~mm}$ \\
\hline number tested & 6 & 2 & 1 \\
best RMS WFE & $0.60 \mathrm{~nm}$ & $0.69 \mathrm{~nm}$ & $0.55 \mathrm{~nm}$ \\
\hline
\end{tabular}

a Track length is the distance between the object and image planes. b RMS wavefront error (WFE) based on a 37-term Zernike polynomial fit. For each class of lens, these are the lowest observed values. 


\section{VISIBLE-LIGHT INTERFEROMETRY}

A visible-light interferometry principle called the Sommargren interferometer or the phase-shifting diffraction interferometer (PSDI) ${ }^{20,21}$ has been used to characterize EUV optics with sub-nanometer accuracy and angstrom-scale precision. ${ }^{22,23}$ In this technique a short-coherence-length laser is used with a beam splitter and a delay line to launch a pair of temporally coherent beams, with a time delay, into an optical fiber.

In the single, concave mirror testing arrangement, for example, the beams emerge from the fibers and are focused onto a spatial-filter pinhole buried within a mirror placed near the mirror's radius of curvature. A small pinhole size is selected to fill the aperture of the mirror with half of the diffracted beam; while the second half of the beam propagates to a CCD camera, without re-imaging optics (to avoid the contribution of additional aberrations). In this way, the pinhole diffracted light serves as a spherical illumination beam for the mirror under test and as a reference beam for interference at the CCD. The time delay is set to allow the test beam to propagate to the mirror and back; the two beams coincide and propagate together to the CCD where their interference is measured.

In the system-measuring configuration, the time-delayed pulses are launched into a pair of fibers destined for spatial-filter pinholes in the object and image planes. In the lower-NA object side, the same pinhole and mirror configuration is used as in the single-mirror test, but rather than have the beam reflect from the test mirror and return, the test and reference beams are supplied by the two pinholes independently. After traversing the optic, the test beam is reflected from the mirror containing the reference beam's pinhole, and the two beams propagate to the CCD where their interference is measured.

In both the single-mirror and the system tests, after interferometrically measuring the test wave's amplitude and phase in the CCD plane, mathematical algorithms are used to numerically propagate the measured wave back to the surface of the mirror, or to the optic's exit pupil plane, effectively undoing the diffraction effects of lensless propagation. Polarization control and careful systematic error calibrations ${ }^{23-25}$ have become central elements of achieving ultra-high accuracy. Throughout the development of visible-light system-measuring interferometers suitable for testing EUV optics, careful comparison with EUV interferometric measurements of the same optical systems have provided an important feedback mechanism for improving the measurement accuracy. 22,23

\section{EUV INTERFEROMETRY}

In 1996, an experimental facility for at-wavelength EUV interferometry was created at LBNL's Advanced Light Source (ALS) beamline 12.0.1. ${ }^{26}$ This undulator beamline is optimized for high coherent flux, delivering approximately $10 \mu \mathrm{W}$ at $13-\mathrm{nm}$ wavelength $(\lambda / \Delta \lambda=200)$ through a $0.5 \mu \mathrm{m}$ pinhole at the beamline's focal point. Both the wavelength and the spectral bandwidth are tunable from $\lambda=6-20-\mathrm{nm}$, and $\lambda / \Delta \lambda$ from 55-10,000. Experimental endstations have been installed on two branches of this beamline to measure and align EUV lenses with numerical aperture values from 0.08 to 0.30 ( $f / 6.25$ to $f / 1.67)$. Optics are measured in their design orientation.

In our measurements of EUV optics, two interferometer configurations (Fig. 1) have proven to be most successful: the phase-shifting point-diffraction interferometer $(\mathrm{PS} / \mathrm{PDI})^{27}$ and the cross-grating lateral-shearing interferometer (LSI) ${ }^{28}$; each design has specific advantages and disadvantages. In addition to these interferometers, a prototype EUV Hartmann wavefront sensor has been operated successfully at low NA. ${ }^{29}$ As implemented in our laboratory, these interferometers measure wavefront aberrations in two dimensions.

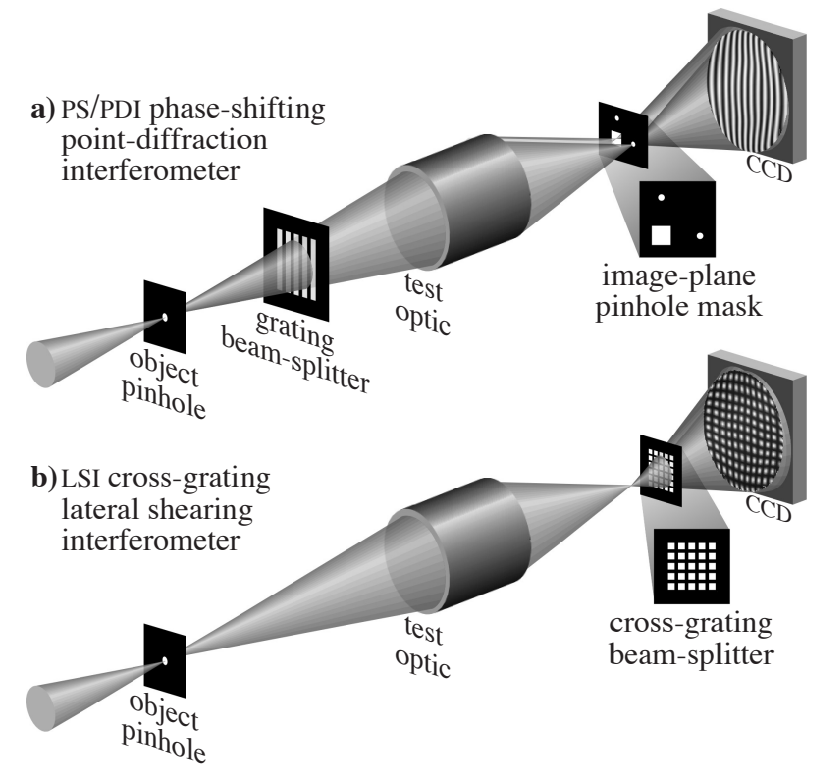

FIG 1. Schematic drawings showing the essential optical elements of the (a) PS/PDI and the (b) LSI interferometers. Light is incident from the left. 


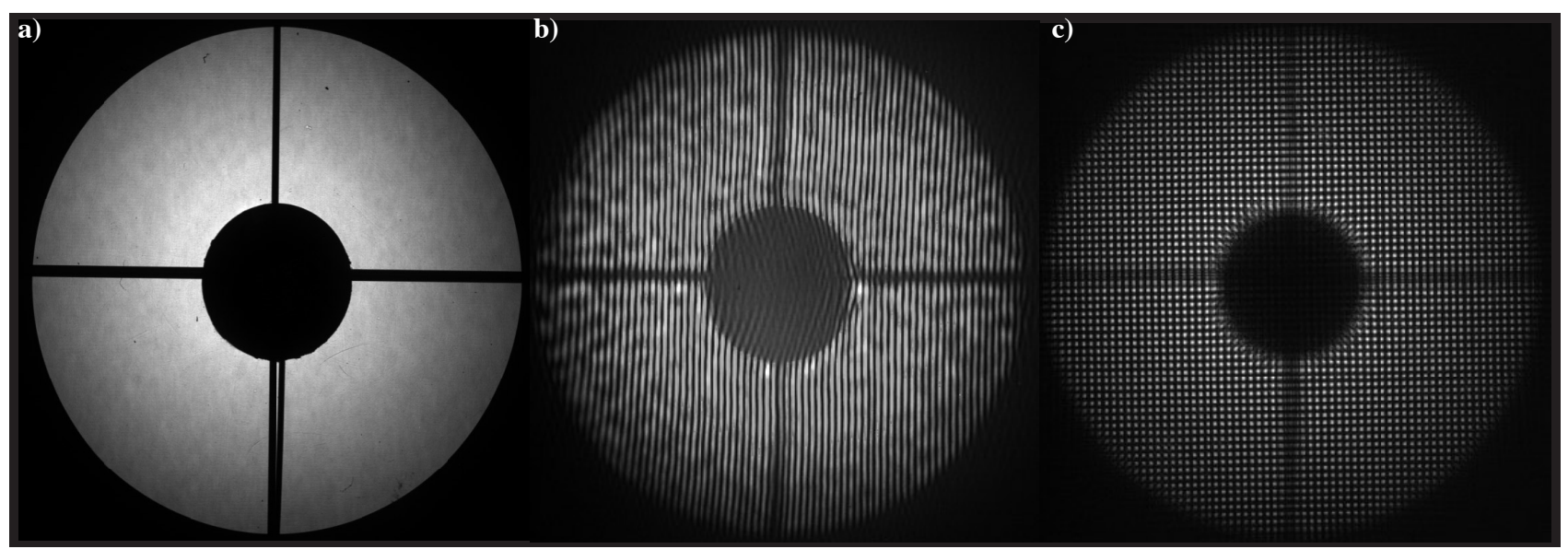

FIG. 2. Data recorded during interferometry of the 0.3-NA MET optic. (a) Light from the annular pupil projected onto the CCD. Thin spiders support a baffle used to block straight-through light. (b) PS/PDI interferogram. (c) LSI interferogram.

Unlike many conventional interferometer designs, these interferometers include no re-imaging optics, and none are required: light propagates to a detector placed in the far-field region. This lensless configuration removes the significant potential for errors introduced by aberrations or distortion from re-imaging optics. However, geometric path length differences between the test and reference beams can be significantly larger than the aberrations of interest. Therefore careful null testing is required to account for systematic, subtractable measurement errors. Furthermore, a radial distortion introduced by the projection of spherical waves onto a planar detector must be accounted for in the data analysis. Figure 2 shows three images recorded by an EUV-sensitive CCD during interferometry.

5.1 The PS/PDI. The PS/PDI ${ }^{23,27,30-32}$ (Fig. 1a) has become the accuracy standard for EUV optical system measurements conducted in our laboratory. Pinhole diffraction is used to create spherical reference waves of exceptionally high quality. An object-plane pinhole illuminates the entrance pupil; in the image plane, a second pinhole in an opaque membrane with open-stencil features produces a spherical reference wave that interferes with the aberrated test beam. A nearby window in the image-plane mask transmits the low-spatial-frequency components of the test beam without attenuation. A PS/PDI interferogram is shown in Fig. 2b.

As a rule of thumb, the required pinhole sizes are comparable to or smaller than the focal spot size of the optical systems under test. For the MET optic (with $0.3 \mathrm{NA}$ ), this requirement translates to 25-35-nm diameter pinholes. Even at this small size, the pinholes must be of high quality, and must be opaque in the vicinity of the pinhole. We nanofabricate pinholes in 100-150-nm-thick free-standing Ni substrates using electron-beam lithography on LBNL's Nanowriter. ${ }^{33}$ The trade-off between the pinhole size (and hence its transmission) and the quality of the test beam is a persistent concern. The desire for high-quality reference waves leads to the selection of smaller pinhole sizes where the relative transmission can be low. In order to match the intensities of the test and reference beams, and maintain high fringe contrast for a good signalto-noise ratio in the measurement, the grating beam-splitter's duty cycle (fraction of open area) can be adjusted. ${ }^{34,35}$ The amount of light transmitted through the pinhole also has a strong dependence on the alignment state and the quality of the optical system under test. As alignment or optical quality improves, the Strehl ratio approaches unity; and because the peak intensity at focus determines the amount of light available for the reference beam's pinhole transmission, the measurement becomes easier to perform. It follows that the PS/PDI can only be used effectively where aberration magnitudes are small (below $\lambda / 2$, perhaps).

5.2 The LSI. The LSI (Fig. 1b) has a number of advantages that make it well suited to the alignment of short-wavelength optical systems where aberration magnitudes are modest (but can be much larger than in the PS/PDI). There are a number of different LSI configurations ${ }^{36}$; we chose to use a relatively simple one. Compared with the PS/PDI, LSI alignment is particularly easy. The LSI requires an object-plane entrance pinhole (like the PS/PDI), and a single grating placed in a Talbot plane close to the image-plane (before or after). Talbot plane distances are given approximately by $z \approx \pm n d^{2} / \lambda,{ }^{28}$ where $z$ is the longitudinal grating displacement from focus, $d$ is the grating pitch, $\lambda$ is the wavelength, and $n$ is an integer. 
When the grating occupies a Talbot plane, and the aberrations are relatively small, a clear, high-contrast fringe pattern is projected onto the CCD. Multiple diffraction orders from the grating overlap with small relative displacements (i.e. shear). The resultant interference pattern can be analyzed to extract the wavefront gradient in the direction of the shear. Using a cross-grating, we project a two-dimensional fringe pattern and collect a pair of orthogonal gradient measurements at the same time. Performing the two directions of measurement simultaneously, instead of sequentially with different or rotated gratings, removes uncertainty in the astigmatism measurement (among other aberrations).

Analysis of the shearing data involves reconstruction of the test wavefront and can be challenging to perform with high precision and accuracy. The Fourier-transform method of interferogram analysis ${ }^{37}$ (with or without phase-shifting) is used to selectively extract derivative measurements in the two directions. Reconstruction can be performed with a local leastsquares minimization algorithm ${ }^{38}$ or a singular value decomposition (SVD) algorithm ${ }^{39,40}$ based on derivatives of the aberration polynomials. Especially important in high-NA measurement, care must be taken to ensure that any variations in the shear magnitude caused by the changing angle of incidence across the grating are accounted for in the analysis.

By adjusting the grating pitch, the shear magnitude and hence the sensitivity of the interferometer can be adjusted. The wavefront is calculated only over the sub-domain of the pupil where multiple-beam overlap occurs. This excludes areas near any domain boundary, including spiders and a central obscuration if present, and the outer edges of the pupil. While a large shear value increases the sensitivity of the measurement a larger fraction of the pupil must be excluded. Our interferometer designs typically include several different cross gratings, with different pitch values, arranged so the appropriate grating can be selected. In our experience with nearly-diffraction-limited EUV optics, ratios of the shear angle, $\sin ^{-1} \lambda / d$, to the NA angle in the $1-5 \%$ range form a good compromise to the above considerations.

Besides the uncertainties introduced by noise-sensitivity in the reconstruction of the test wavefront from its measured gradients, one significant complication for high-accuracy shearing comes from noise limitations in the measurement. In some cases, the accurate measurement of a given aberration requires a much more accurate measurement of its derivative aberrations.

\section{ACCURACY}

Accuracy is the critical characteristic of all wavefront-measuring interferometers. While precision comes from system stability and high signal-to-noise ratio, achieving high measurement accuracy requires careful calibration. The extension of these techniques from low NA to higher NA values leads to orders of magnitude higher difficulty in maintaining accuracy. Many of the compensable systematic error contributions scale as powers of the NA value and can become significantly larger than the wavefront aberrations magnitudes of interest.

We use a series of null tests to characterize the various elements of the interferometers, including the geometry of measurement. The simplicity of the interferometer designs and the small number of optical elements (pinholes, gratings, and CCD) reduces the complexity of the calibrations compared with other interferomtery schemes. However pinhole diffraction to high NA is a significant challenge for EUV interferometry, and characterizing the measurement geometry in the absence of re-imaging optics poses a serious challenge. In addition to null-tests and other in situ measurements, we rely on crosscalibration among different interferometry methods; and ultimately we derive proof of the accuracy of our measurements from high-resolution imaging tests (i.e. lithographic printing in photoresist.)

6.1 Pinhole-diffracted reference waves. The PS/PDI and LSI EUV interferometers are dependent on pinhole diffraction to produce spherical reference waves. Although the detailed shape profiles of our sub-40-nm pinholes are difficult to ascertain experimentally, the complex dependencies of pinhole size and thickness and reference wave quality have been studied through experiments and modeling. In this size domain, scalar diffraction theory fails to predict the pinhole transmission efficiency, polarization dependence, or wavefront quality, among other effects. Adding to the complexity is the fact that the pinholes filter, but do not entirely remove, the aberrations in the wavefronts incident upon them. We have compared angle-resolved diffraction measurements, conducted on arrays

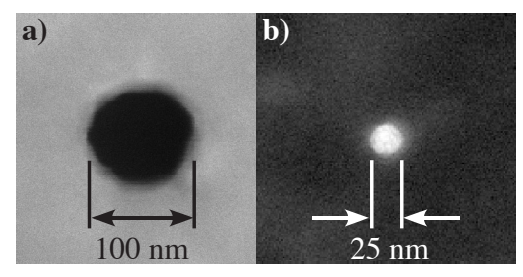

FIG 3. Free-standing Ni-membrane pinholes used to create spherical reference waves. (a) SEM micrograph of an object-side pinhole. (b) TEM micrograph of an image-side pinhole. 


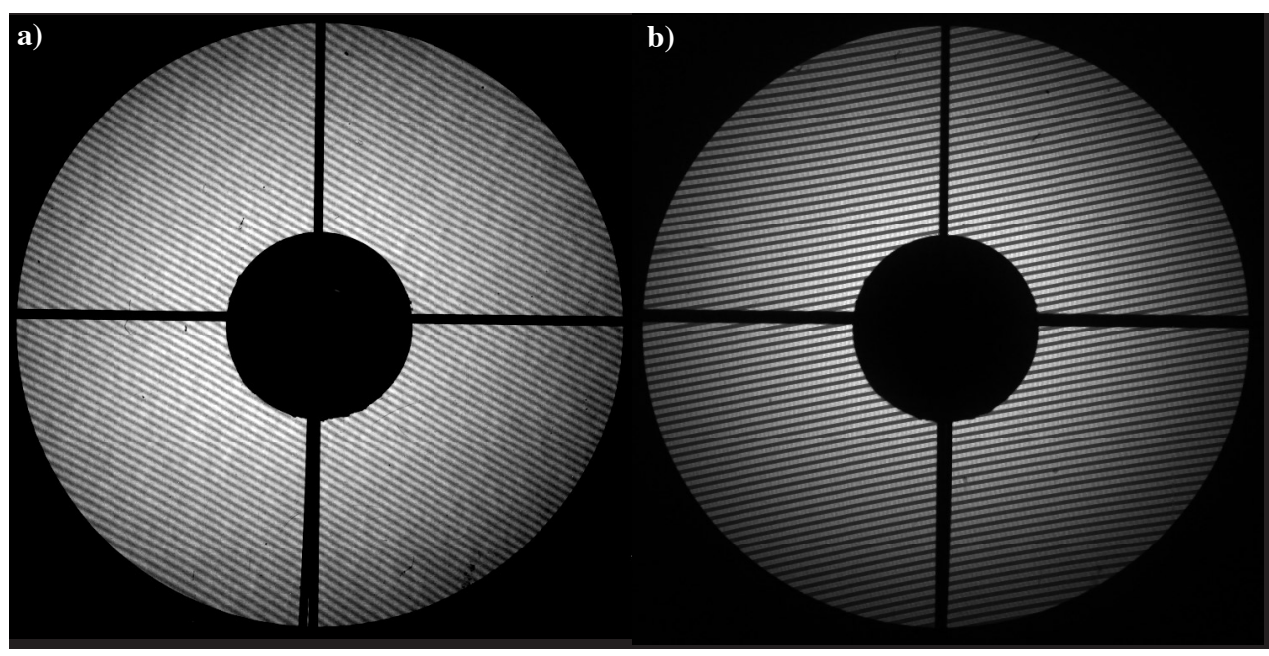

FIG 4. Null-test interferograms recorded during the calibration of the 0.3-NA MET interferometer. (a) Two-pinhole diffraction pattern from closely-spaced pinholes in the object plane. (b) Projected fringe pattern with the PS/PDI grating inserted in the beam, with no image-plane spatial filtering.

of nominally identical pinholes, with modeling performed using TEMPEST-3D, a finite-difference time-domain (FDTD) vector electromagnetic-field simulation program. ${ }^{41}$ A challenging aspect of using the PS/PDI is aligning the reference beam onto the image-plane pinhole - both the lateral and longitudinal alignments are critical. Experimentally, we use arrays of patterned alignment features and a variety of pinhole sizes so that pinholes with the ideal size can be found and used. ${ }^{14,42}$ Finally, we perform measurements with a number of nominally identical pinholes in order to reduce the effects of individual pinhole irregularities through averaging.

6.2 Systematic error calibrations. A number of tests have been developed for the measurement of systematic errors in the lensless interferometers described here. By removing the pinholes from the image-plane in the PS/PDI, the quality and alignment of the beam-splitter grating (Fig. 4a) can be measured. Using two, closely-spaced pinholes in the object plane (Fig. 4b) or the image-plane we perform two-pinhole null tests. These tests allow us to calibrate NA of measurement; and by performing the tests with multiple pairs of pinholes, we obtain statistical information about the spherical quality of the diffracted waves. Other tests have been developed to characterize the measurement geometry, including the precise position and angle of the CCD detector, which can contribute systematic errors if it is not well characterized.

6.3 Intercomparison. The intercomparison of different wavefront measurement methods applied to the same optical system provides invaluable feedback on the relative strengths and weaknesses of the different techniques. We have performed visible-light and EUV interferometry cross-comparison tests on nine individual EUV optical systems. These optics were transported from LLNL to LBNL and back, maintaining their alignment state to a fraction of a nanometer (in the measured wavefront) in all cases. Figure 5 shows intercomparisons of visible-light and various EUV measurements of two different optics. ${ }^{14,22}$ Through these direct comparisons, EUV interferometry has been used to identify and remediate systematic error sources in the visible-light interferometers. Furthermore, specific differences in the PS/PDI and LSI measurements are being used to improve the accuracy of the LSI technique. Despite careful comparisons, some differences still remain, primarily concentrated in the low-spatial frequency aberrations such as astigmatism, coma, and spherical aberration. Between the visible-light and EUV measurements, the highest level of agreement achieved to date has been on the order of $0.25-\mathrm{nm}$ RMS in a 37-term Zernike polynomial series. ${ }^{43,44}$ Among the
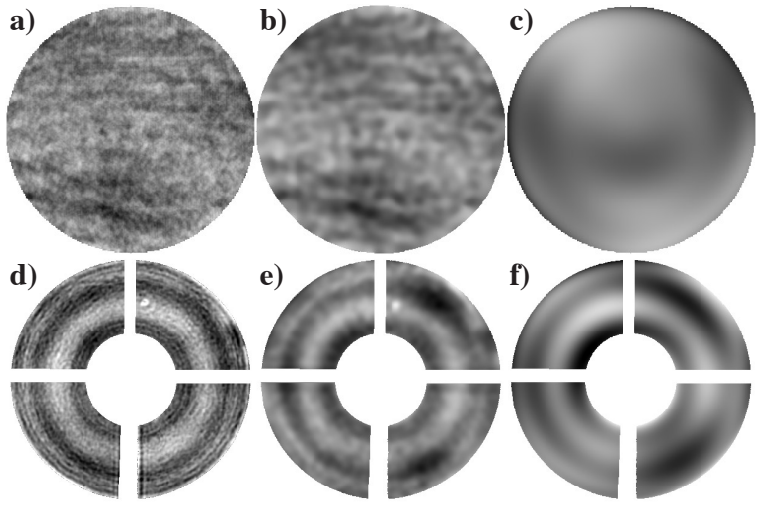

f)

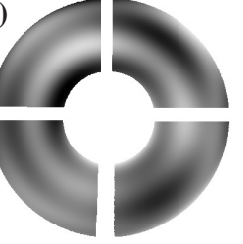

FIG 5. Intercomparison of measured wavefronts: (a-c) are from the 0.1-NA ETS optic, (d-f) are from the 0.3-NA MET. (a) Visible-light; (b) EUV; (c) difference wavefront filtered to include only 37 Zernike terms. (d) Visible-light; (e) EUV PS/PDI; (f) EUV LSI. Note that each interferomter has a different spatial-frequncy cutoff; only low-spatial-frequencies are used for alignment, and all interferometers capture well beyond the 37-term Zernike series. [Refs. 14, 44] 

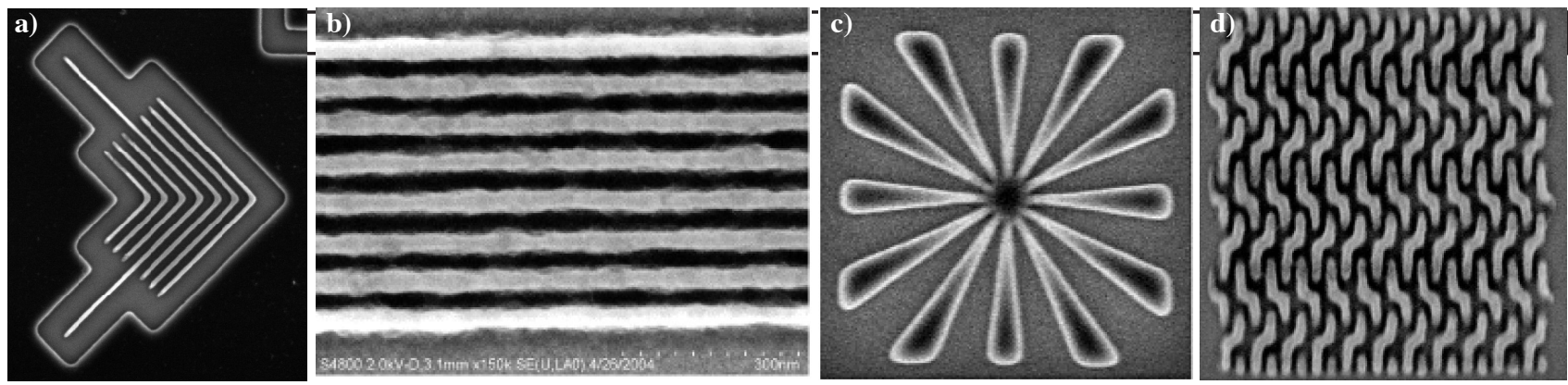

FIG. 6. Images printed in photoresist, created with EUV lithography. (a) Printed with the 0.1-NA ETS optic in Rohm and Haas EUV-2D photoresist, this pattern has $39-\mathrm{nm}$ linewidths, $160-\mathrm{nm}$ pitch; lines were narrowed by $1.4 \times$ overexposure. This image, and a series of images throgh focus, shows no evidence of astigmatism. Various test patterns printed with the 0.3-NA MET in Rohm and Haas MET-1K photoresist; (b) 30-nm half-pitch; (c) 1.8- $\mu$ m-wide sector star pattern; (d) circuit pattern with 35-nm features. [Refs. 45-47]

different EUV techniques, the level of agreement is closer to 0.1-nm RMS. Similar levels of agreement have been achieved by EUVA researchers in Japan. ${ }^{36}$

6.4 Imaging. For projection lithography optics, the ultimate validation of interferometry and alignment accuracy comes from imaging experiments in which high-resolution patterns are printed in photoresist. Figure 6 shows some of the highestresolution images ever recorded with optical projection lithography. ${ }^{45-47}$ Detailed, quantitative wavefront measurements are used to predict imaging behavior. Currently the limitations in this technique come from the resolution limits of modern (experimental) EUV photoresists, which are not yet able to resolve at the length scales of diffraction-limited performance in the highest-resolution EUV optics (resist limitations are presently in the 25-35-nm range. ${ }^{48}$ ) Similar validation for telescopes would come from the ability to resolve small, faint objects.

\section{CONCLUSIONS}

Ultra-high-accuracy mirror fabrication, coating and testing-metrology techniques now exist to meet the requirements of advanced, diffraction-limited, reflective EUV optical systems. The semiconductor industry's plans to use 13-nm-wavelength EUV lithography for generations of micro-electronics fabrication with feature half-pitch below $45 \mathrm{~nm}$ have spurred the development of the worlds highest resolution light-projection imaging systems. According to industry timetables, ${ }^{1}$ these systems, and their accompanying fabrication and testing technologies, will be ready for mass production sometime between 2009 and 2013. Where new space telescope projects demand ever-higher optical quality and higher-angular resolution, the astronomy community can take advantage of these new developments to set significantly more stringent optical specifications than before. One-to-two angstrom-scale figure and finish tolerances with sub- $\mu$ rad slope errors are achievable now. Presently, several of the eight wavelength channels on the four-telescope AIA instrument ${ }^{4}$ on the Solar Dynamics Observatory (being prepared for launch in 2008) already utilize multilayer coating technology that was developed to meet the optical requirements of EUV lithography ${ }^{13}$; however those telescopes do not require the same tight figure specifications as EUV lithography optics.

The key to achieving such high quality optical systems is metrology. In collaboration with the semiconductor industry and national laboratory partners in the US, we have developed interferometers with sub-angstrom wavefront measuring accuracy. Over the past ten years, we have successfully measured and aligned nine EUV optical systems, adhering to ultra-high vacuum (UHV) cleanliness, and cleanroom assembly guidelines required for EUV lithography. We use visible-light interferometry to characterize single mirror elements and to align the assembled systems; and we use various EUV interferometry configurations and calibration methods to align and characterize completed lenses with ultra-high-accuracy. Claims of ultra-high accuracy are supported by photoresist imaging experiments conducted at LBNL.

We believe that these methods can and should be adapted to the fabrication and testing of astronomical EUV telescopes. Furthermore, newly developed coherent EUV sources may free these metrology techniques from their dependence on synchrotron sources. 


\section{ACKNOWLEDGMENTS}

This work would not be possible without the expert contributions of LBNL's Center for X-Ray Optics members Kevin Bradley, Brian Hoef, René Delano, Ronald Oort, C. Drew Kemp, M. Gideon Jones, Ronald Tackaberry, and Robert Gunion; and from LLNL, Carl Chung, Layton Hale, Nhan Nguyen, and Donald Sweeney. This work is funded by International Sematech, the EUV Limited Liability Corporation (EUV LLC), and by the U.S. Department of Energy. Parts of this work were performed under the auspices of the U.S. Department of Energy by University of California, Lawrence Berkeley National Laboratory, and by Lawrence Livermore National Laboratory under contract No. W-7405-Eng-48.

\section{REFERENCES}

1. International Technology Roadmap for Semiconductors (ITRS), http://www.itrs.net/Common/2004Update/2004_07_Lithography.pdf

2. http://www.intel.com/pressroom/archive/releases/20040802tech.htm, and http://www.intel.com/pressroom/archive/releases/20040225corp_b.htm.

3. Information on the Chandra X-Ray Observatory, http://chandra.harvard.edu.

4. Information on the AIA mission, http://aia.Imsal.com.

5. K. A. Goldberg, "Extreme Ultraviolet Interferometry," Doctoral Dissertation, Physics Department, Univ. Calif. Berkeley, 1997, p. 271.

6. D. Attwood, G. Sommargren, R. Beguiristain, K. Nguyen, J. Bokor, N. Ceglio, K. Jackson, J. Underwood, "Undulator radiation for at-wavelength interferometry of optics for extreme-ultraviolet lithography," Appl. Opt. 32 (34), 7022-31 (1993).

7. R. Beguiristain, K. A. Goldberg, E. Tejnil, "Interferometry using undulator sources," Rev. Sci. Instrumen. 67 (9), 3353 (1996)

8. M. Niibe, M. Mukai, T. Tanaka, K. Sugisaki, Y. Zhu, Y. Gomei, "Development of EUV point diffraction interferometry using the NewSUBARU undulator radiation," Proc. SPIE 4782, 204-11 (2002).

9. One notable exception is: A. K. Ray-Chaudhuri, K. D. Krenz, R. P. Nissen, S. J. Haney, et al., "Initial results from an extreme ultraviolet interferometer operating with a compact laser plasma source,” J. Vac. Sci. \& Technol. B 14 (6), 3964-68 (1996).

10. E. L. Antonsen, K. C. Thompson, M. R. Hendricks, D. A. Alman, B. E. Jurczyk, D. N. Ruzic, T. D. Chinh, G. Edwards, S. Wurm, O. Wood, R. Bristol, "XCEED: XTREME commercial EUV exposure diagnostic experiment," Proc. SPIE 5751, 1192-1202, (2005).

11. H. C. Kapteyn, M. M. Murnane and I. P. Christov, "Coherent X-Rays from Lasers: Applied Attosecond Science”, Physics Today, p. 39 (March 2005).

12. J. J. Rocca, Y. Wang, M. A. Larotonda, B. M. Luther, M. Berrill, D. Alessi "Saturated $13.2 \mathrm{~nm}$ high repetition rate laser in nickellike cadmium," Opt. Lett., (2005), in press.

13. R. Soufli, D. L. Windt, J. C. Robinson, E. A. Spiller, F. J. Dollar, A. L. Aquila, E. M. Gullikson, B. Kjornrattanawanich, J. F. Seely, L. Golub, "Development and testing of EUV multilayer coatings for the atmospheric imaging assembly instrument aboard the Solar Dynamics Observatory," Proc. SPIE 5901-24 (2005), in press.

14. K. A. Goldberg, P. Naulleau, P. Denham, S. B. Rekawa, K. Jackson, E. H. Anderson and J.A. Liddle, "At-Wavelength Alignment and Testing of the 0.3 NA MET Optic," J. Va. Sci. \& Technol. B 22 (6), 2956-61 (2004).

15. C. Montcalm, J.A. Folta, C. C. Walton, "Method and system using power modulation and velocity modulation producing sputtered thin films with sub-angstrom thickness uniformity or custom thickness gradients," U.S. Patent No. 6,668,207, December 23, 2003.

16. E. M. Gullikson, S. L. Baker, J. E. Bjorkholm, J. Bokor, K. A. Goldberg, J. E. M. Goldsmith, C. Montcalm, P. Naulleau, E. A. Spiller, D. G. Stearns, J. S. Taylor, J. H. Underwood, "EUV scattering and flare of 10x projection cameras," Proc. SPIE 3676, 717-23 (1999).

17. P. Naulleau, K. Goldberg, E. Gullikson, and J. Bokor, "At-wavelength, system-level flare characterization of EUV optical systems," Appl. Opt. 39 (17), 2941-47 (2000).

18. R. Soufli, E. Spiller, M. A. Schmidt, J. C. Davidson, R. F. Grabner, E. M. Gullikson, B. B. Kaufmann, S. L. Baker, H. N. Chapman, et al., "Multilayer optics for an extreme ultraviolet lithography tool with $70 \mathrm{~nm}$ resolution", Proc. SPIE 4343, 51-59 (2001).

19. D. A. Tichenor, A. K. Ray-Chaudhuri, S. H. Lee, H. N. Chapman, W. C. Replogle, K. W. Berger, R. H. Stulen, G. D. Kubiak, L. E. Klebanoff, J. B. Wronosky, et al., "Initial Results from the EUV Engineering Test Stand," Proc. SPIE 4506, 9-18 (2001).

20. H. N. Chapman, D. W. Sweeney, "Rigorous method for compensation selection and alignment of microlithographic optical systems," Proc. SPIE 3331, 102-13 (1998).

21. G. E. Sommargren, "Phase shifting diffraction interferometer," U.S. Patent 5,548,403, (1996).

22. K. Goldberg, P. Naulleau, J. Bokor and H. Chapman, “Testing EUV Optics with Visible-Light and EUV Interferometry," J. Vac. Sci. \& Technol. B 20 (6), 2834-39 (2002).

23. K. A. Goldberg, P. Naulleau, P. Denham, S. B. Rekawa, K. Jackson, J. A. Liddle, E. H. Anderson, "EUV interferometric testing and alignment of the 0.3-NA MET optic," Proc. SPIE 5374, 64-73 (2004).

24. M. A. Johnson, D. W. Phillion, G. E. Sommargren, T. A. Decker, J. S. Taylor, Lawrence Livermore National Lab.; Y. Gomei, S. Takeuchi, O. Kakuchi, "Construction and testing of wavefront reference sources for interferometry of ultra-precise imaging systems," Proc. SPIE 5869-28, (2005), in press.

25. D. W. Phillion, G. E. Sommargren, M. A. Johnson, T. A. Decker, J. S. Taylor, "Calibration of symmetric and non-symmetric errors for interferometry of ultra-precise imaging systems," Proc. SPIE 5869-30 (2005), in press. 
26. Information about ALS Beamline 12.0.1, http://www-als.lbl.gov/als/techspecs/bl12.0.1.html

27. H. Medecki, E. Tejnil, K. A. Goldberg, and J. Bokor, Opt. Lett. 21, 1526-28 (1996).

28. P. P. Naulleau, K. A. Goldberg, and J. Bokor, "Extreme ultraviolet carrier-frequency shearing Interferometry of a lithographic fourmirror optical system,” J. Vac. Sci. \& Technol. B 18, 2939-43 (2000).

29. P. Mercère, M. Idir, P. Zeitoun, X. Levecq, G. Dovillaire, S. Bucourt, D. Douillet, K. A. Goldberg, P. P. Naulleau, and S. Rekawa , "X-ray Wavefront Hartmann Sensor," AIP Conf. Proc. 705, 819-822 (2004).

30. K. A. Goldberg, E. Tejnil, S. H. Lee, H. Medecki, D. T. Attwood, K. H. Jackson, J. Bokor, "Characterization of an EUV Schwarzschild objective using phase-shifting point diffraction interferometry," Proc. SPIE 3048, 264-70 (1997).

31. P. P. Naulleau, K. A. Goldberg, S. H. Lee, C. Chang, D. Attwood, and J. Bokor, "Extreme-ultraviolet phase-shifting point-diffraction interferometer: a wave-front metrology tool with subangstrom reference-wave accuracy," Appl. Opt. 38 (16), $7252-63$ (1999).

32. K. A. Goldberg, P. Naulleau, P. J. Batson, P. Denham, J. Bokor, J., H. N. Chapman, "EUV Interferometry of a Four-Mirror RingField EUV Optical System," Proc. SPIE 3997, 867-73 (2000).

33. E. H. Anderson, D. Olynick, B. Harteneck, E. Veklerov, et al., "Nanofabrication and diffractive optics for high-resolution x-ray applications," J. Vac. Sci. \& Technol. B 18 (6), 2970-75 (2000).

34. K. A. Goldberg, "Extreme Ultraviolet Interferometry," op. cit., p. 52.

35. P. Naulleau, K. A. Goldberg, E. Tejnil, "Phase-shifting point diffraction interferometer grating designs,” U.S. Patent No. 6,195,169 (2001).

36. K. Sugisaki, M. Okada, Y. Zhu, K. Otaki, Z. Liu, J. Kawakami, M. Ishii, J. Saito, K. Murakami, M. Hasegawa, C. Ouchi, S. Kato, T. Hasegawa, A. Suzuki, M. Niibe, M. Takeda, "Comparison with EUV at-wavelength metrologies," Proc. SPIE 5921-15 (2005), in press.

37. D. J. Bone, H.-A. Bachor, R. J. Sandeman, "Fringe-pattern analysis using a 2-D Fourier transform," Appl. Opt. 25, 1653-60 (1986).

38. M. P. Rimmer, "Method for evaluating lateral shearing interferograms," Appl. Opt. 13, 623-29 (1974).

39. G. Harbers, P. J. Kunst, G. W. R. Leibbrandt, "Analysis of lateral shearing interferograms by use of Zernike polynomials," Appl. Opt. 35 (31), 6162-72 (1996).

40. H. van Brug, "Zernike polynomials as a basis for wave-front fitting in lateral shearing interferometry," Appl. Opt. 36 (13), $2788-90$ (1997).

41. TEMPEST-3D is developed by the A. Neureuther group, Department of EECS, Univ. Calif. Berkeley.

42. K. A. Goldberg, P. Naulleau, P. Batson, P. Denham, H. Chapman, and J. Bokor, "Extreme ultraviolet alignment and testing of a four mirror aspheric extreme ultraviolet optical system," J. Vac. Sci. \& Technol. B 18, 2911-15 (2000).

43. K. A. Goldberg, P. Naulleau, S. H. Lee, C. Chang, C. Bresloff, R. Gaughan, H. N. Chapman, J. Goldsmith, and J. Bokor, "Direct comparison of EUV and visible-light interferometries,"Proc. SPIE 3676, 635-42 (1999).

44. K. A. Goldberg, P. Naulleau, J. Bokor, and H. N. Chapman, "Honing the accuracy of extreme ultraviolet optical system testing: at-wavelength and visible-light measurements of the ETS Set-2 projection optic," Proc. SPIE 4688, 329-37 (2002).

45. P. P. Naulleau, K. A. Goldberg, E. H. Anderson, J. Bokor, B. D. Harteneck, K. H. Jackson, D. L. Olynick, F. Salmassi, S. L. Baker, P. B. Mirkarimi, E. A. Spiller, C. C. Walton, D. J. O’Connell, P. Yan, G. Zhang, "Static EUV microexposures using the ETS set-2 optics," Proc. SPIE 5037, 36-46 (2003).

46. P. Naulleau, K. A. Goldberg, E. H. Anderson, J. Bokor, B. Harteneck, K. Jackson, D. Olynick, F. Salmassi, S. Baker, P. Mirkarimi, E. Spiller, C. Walton, D. O'Connell, P.-Y. Yan, G. Zhang, "Printing-based performance analysis of the engineering test stand set-2 optic using a synchrotron exposure station with variable sigma," J. Vac. Sci. \& Technol. B 21 (6), 2697-2700 (2003).

47. P. P. Naulleau, K. A. Goldberg, E. H. Anderson, J. P. Cain, P. Denham, B. Hoef, K. Jackson, A.-S. Morlens, S. Rekawa, K. Dean, "EUV microexposures at the ALS using the 0.3-NA MET projection optics," Proc. SPIE 5751, 56-63 (2005).

48. P. Naulleau, K. A. Goldberg, J. P. Cain, E. Anderson, P. Denham, K. Jackson, B. Hoef, S. Baker, E. Spiller, K. Dean, "Characterization of the Synchrotron-based 0.3-NA EUV Microexposure Tool at the ALS," J. Vac. Sci \& Technol. B 23 (6), (2005), to be published. 